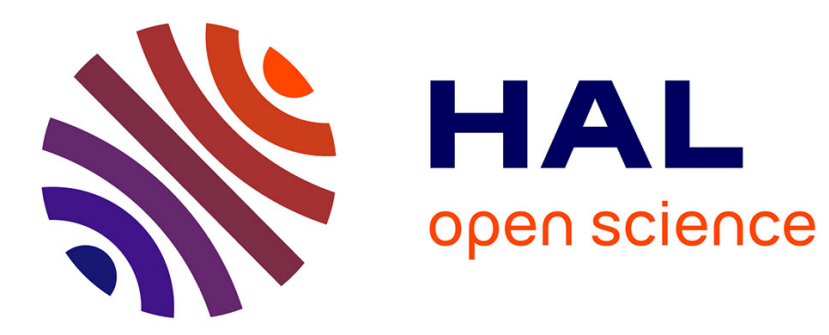

\title{
Le théâtre antique
}

Jean-Yves Marc, Pierre Mougin, Séverine Blin, Nathalie André

\section{To cite this version:}

Jean-Yves Marc, Pierre Mougin, Séverine Blin, Nathalie André. Le théâtre antique. Gallia - Archéologie de la France antique, 2007, Epomanduodurum, une ville chez les Séquanes: bilan de quatre années de recherche à Mandeure et Mathay (Doubs), 64, pp.396-407. 10.3406/galia.2007.3321 . hal01911687

\section{HAL Id: hal-01911687 \\ https://hal.science/hal-01911687}

Submitted on 7 Feb 2020

HAL is a multi-disciplinary open access archive for the deposit and dissemination of scientific research documents, whether they are published or not. The documents may come from teaching and research institutions in France or abroad, or from public or private research centers.
L'archive ouverte pluridisciplinaire HAL, est destinée au dépôt et à la diffusion de documents scientifiques de niveau recherche, publiés ou non, émanant des établissements d'enseignement et de recherche français ou étrangers, des laboratoires publics ou privés.

\section{(이) $\$$}

Distributed under a Creative Commons Attribution - NonCommercial - NoDerivatives 44.0 


\section{LE THÉÂTRE ANTIQUE}

Les théâtres des provinces occidentales de l'Empire romain souffrent, encore aujourd'hui, d'un manque criant de publications monographiques. Parmi eux, les édifices de spectacle des Gaules ou des Germanies sont encore plus mal servis par la recherche (voir les remarques de P. Gros in Dumasy, 2000, p. 11). Enfermés dans une catégorie typologique, celle du "théâtre gallo-romain », qui pose en réalité plus de problèmes qu'elle n'en résout, ils n'ont que très rarement fait l'objet d'études architecturales exhaustives. Des publications récentes, comme à Lenzburg en Suisse (Niffeler, 1988) ou à Argentomagus/Saint-Marcel en France (Dumasy, 2000), ou des recherches en cours, à Alésia ou à Orange, viennent heureusement commencer à combler les lacunes considérables qui rendent hasardeuses toutes les conclusions historiques que l'on souhaiterait tirer de ces constructions dont on devine la place primordiale qu'elles occupèrent dans la panoplie monumentale des villes romaines. La reprise des études au théâtre de Mandeure s'est placée d'emblée dans une perspective de publication monographique. C'est dire que ce premier bilan scientifique, rédigé au terme des quatre premières campagnes de fouilles et d'étude, doit être considéré comme provisoire. Des secteurs importants de l'édifice restent peu ou mal connus et bien des points sur lesquels nous travaillons sont encore hypothétiques. Pourtant, nos recherches ont amplement complété ou modifié les travaux de nos prédécesseurs. Les étapes du développement monumental du théâtre de Mandeure, ses caractéristiques morphologiques, l'ampleur et la qualité de son décor architectural en font un monument particulièrement important dans l'architecture théâtrale des provinces du nord-ouest de l'Empire romain : voilà qui justifie la présentation de nos premières conclusions.

Le théâtre antique de Mandeure est appuyé contre le talus calcaire qui borde le Doubs (fig. 2). Cet édifice considérable, de $142 \mathrm{~m}$ de diamètre, comprenait une cavea à quatre volées de gradins (maeniana), en partie appuyée sur le talus naturel, en partie construite sur des substructions en opus vittatum, un dispositif scénique avec orchestra et pulpitum, ainsi que trois façades constituées d'arcades décorées d'un ordre engagé (fig. 21).

\section{HISTORIQUE DES RECHERCHES}

Le théâtre fut abandonné à une date encore inconnue. Un petit bronze de Constantin a été découvert lors de travaux de restauration en 1972 mais, comme le contexte topographique et stratigraphique de cette trouvaille est inconnu, il est impossible d'en tirer parti (Morel, 1974). Ce qui est sûr, en revanche, c'est que le théâtre partage dès le milieu du $\mathrm{IV}^{\mathrm{e}} \mathrm{s}$. le destin de la parure monumentale de l'agglomération antique d'Epomanduodurum et fait l'objet d'une spoliation systématique qui touche principalement les façades en grand appareil et les gradins de la cavea. Les matériaux récupérés sont utilisés dans l'enceinte de la fortification tardive construite devant le pont sur le Doubs (voir infra, p. 407 sq.). La précocité de cette destruction n'est guère étonnante dans la région : à Avenches, c'est même dès 268 que le théâtre est fortifié pour servir de refuge à la population (Matter, 1999). Les ruines de l'édifice de spectacle étaient encore visibles à l'époque mérovingienne, comme l'atteste la découverte d'une sépulture dans un des caissons de l'angle sud-ouest de l'édifice (Tchirakadzé, 1983). Mais, pendant plus de mille ans, le théâtre antique tombe dans l'oubli et la cavea dut se confondre très vite avec le festonnage de la petite falaise calcaire qui longe le Doubs. L'édifice échappa en tous cas aux recherches menées à partir de 1581 à l'initiative du comte Frédéric de Wurtemberg-Montbéliard par Jean Bauhin et surtout par l'architecte et antiquaire Heinrich Schickhardt qui, le premier, identifia le site d'Epomanduodurum et en publia les premières découvertes (Bouvard dir., 2002). On peut s'étonner que des ruines aussi imposantes aient échappé aussi longtemps à la curiosité des antiquaires, d'autant que le lieu-dit est, à l'époque de la Révolution française, "cirque romain " et que le clergé connaissait suffisamment bien les vestiges pour y cacher les objets du culte menacés par la nationalisation des biens de l'Église. Quoi qu'il en soit, l'histoire de la découverte du théâtre ne commence véritablement qu'au début du XIX ${ }^{\mathrm{e}} \mathrm{s}$.

La découverte du théâtre antique de Mandeure constitue très certainement un des chapitres les plus significatifs de la genèse des monuments historiques et de l'histoire de l'archéologie monumentale gallo-romaine (Durand, 2000). C'est dans les premières années du XIX ${ }^{\mathrm{e}}$ s., pendant ces années de la Restauration et de la Monarchie de Juillet qui voient, en France, se développer à la fois une curiosité archéologique grandissante et les premières préoccupations patrimoniales, que F. Morel-Macler (1787-1884), architecte de la ville de Montbéliard, entreprend de dégager les vestiges du théâtre qui affleurent alors à peine. Il met au jour la cavea, l'orchestra et le dispositif scénique. En revanche, les façades en grand appareil ne sont pas reconnues, même si un assez grand nombre d'éléments lapidaires, qui se sont révélés depuis appartenir aux arcades des façades, sont découverts. Vestiges, blocs architecturaux et matériel 


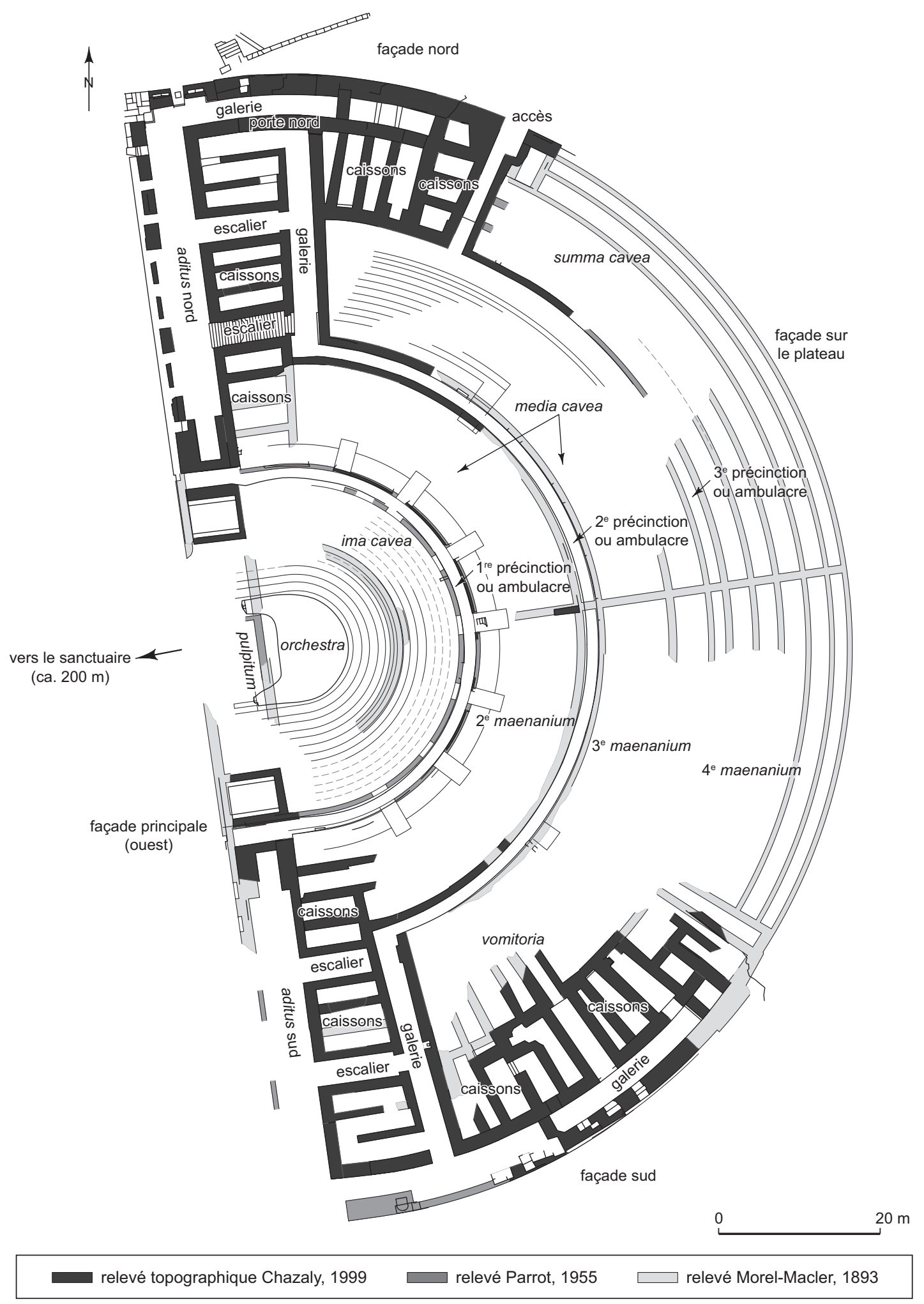

Fig. 21 - Plan schématique du théâtre de Mandeure (DAO : N. André). 


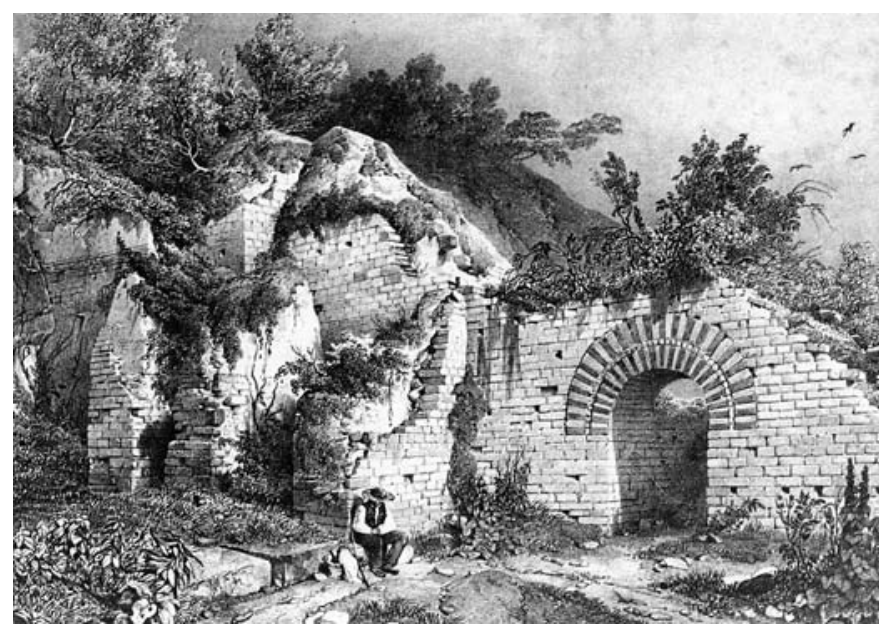

Fig. 22 - Gravure ancienne montrant la porte nord du théâtre de Mandeure (d'après Golbéry, 1828).

découverts lors des fouilles sont rapidement publiés par la Société d'émulation de Montbéliard qui avait été très active dans ces premières fouilles (Morel-Macler, 1847). Les ruines deviennent vite célèbres et figurent en bonne place dans les premiers guides touristiques de la région publiés par C. Nodier ou P. de Golbery (fig. 22), mais les travaux de dégagement sont vite arrêtés, faute de crédits. En juin 1836, Prosper Mérimée, alors inspecteur des Monuments historiques, visite les vestiges du théâtre en compagnie de F. Morel-Macler; il est très impressionné par les vestiges, qu'il range de manière erronée dans « les monuments de la décadence romaine » : «Ce monument dont le patriotisme des antiquaires alsaciens a un peu exagéré l'importance paraît appartenir à une époque très avancée de décadence. Ainsi, rien de classique dans les profils des chapiteaux et des corniches dont on a retrouvé de nombreux débris. Toutefois leur étrangeté même les rend sujet d'étude, car l'histoire de l'art embrasse aussi bien les époques de sa décadence que de sa perfection. L'une des portes du théâtre, celle du Nord, presque intacte, offre une singularité que je n'avais jamais observée auparavant et dont je ne connaissais pas d'exemples antiques. Son archivolte et ses piédroits sont formés de matériaux de deux couleurs différentes et alternant entre elles du grès rouge et du tuffeau blanc. Voilà ce me semble un fait très remarquable et qui montre le passage insensible entre l'architecture romaine et celle du Moyen-Âge ». Inutile d'insister sur les erreurs manifestes de l'auteur dans ses appréciations chronologiques et stylistiques : retenons que son enthousiasme déboucha sur le classement de l'édifice quelques années plus tard, en 1843 (Duvernoy M., 1952). Cette sollicitude administrative resta malheureusement sans lendemain et le théâtre fut laissé à l'abandon pendant de longues années. La situation est d'autant plus surprenante que le théâtre de Mandeure connut très vite une notoriété scientifique : c'est, en effet, un des édifices qui servit à définir le type du théâtre gallo-romain (Caumont, 1862). Mais les ruines du théâtre entrent alors dans une période d'abandon, interrompue par de rares interventions archéologiques, comme celle qui, en 1865, mit au jour le dallage de l'orchestra (Duvernoy, 1873). Quand C. Duvernoy (18181909), professeur au collège de Montbéliard et fondateur de la Société d'émulation de Montbéliard, reprend des fouilles dans l'agglomération antique de Mandeure, il ne s'intéresse que peu au théâtre et se consacre au sanctuaire romain proche. Pendant la longue période d'abandon qui suit, retenons qu'en 1893 les vestiges du théâtre, de même d'ailleurs que ceux du temple, sont relevés et reportés sur le plan cadastral à l'initiative de la Société d'émulation de Montbéliard, qui saisit cette année-là l'occasion d'une importante sécheresse qui avait rendu visibles de nombreux vestiges, ignorés par F. Morel-Macler (fig. 3).

Entre la fin du $\mathrm{XIX}^{\mathrm{e}}$ s. et le milieu du XX ${ }^{\mathrm{e}}$ s., les recherches cessèrent complètement. En 1945, les parties de l'édifice qui avaient été anciennement dégagées n'étaient quasiment plus visibles. La voûte de la porte Nord n'émergeait plus qu'à quelques dizaines de centimètres du sol, alors qu'elle avait été entièrement fouillée par F. Morel-Macler. Les fouilles reprirent en 1946. Cette année-là, L. Lerat, professeur d'archéologie classique à l'université de Franche-Comté et premier directeur régional des Antiquités historiques de Franche-Comté, fait entreprendre des travaux de restauration et de remise en état de l'édifice. En 1951, le service des Ponts et Chaussées, à la recherche de $8000 \mathrm{~m}^{3}$ de remblais pour la reconstruction du pont sur le Doubs entre Mandeure et la commune limitrophe de Mathay, s'entendit avec le service des Antiquités pour prélever le matériau sur le théâtre situé à moins de $300 \mathrm{~m}$ du chantier de construction. Ce sont deux membres de la Société d'émulation de Montbéliard, Marcel Duvernoy et Robert Cuisenier, qui sont chargés de surveiller le suivi archéologique de l'opération. Les fouilles, menées de manière «peu orthodoxe mais décisive », selon l'expression même des fouilleurs, permettent de remettre au jour la cavea et l'orchestra et de dégager l'ensemble de la grande façade diamétrale ouest. De très nombreux éléments architecturaux sont également découverts. Dans les années qui suivent, c'est un professeur du lycée technique de Montbéliard, Paul Parrot, qui poursuit les fouilles avec ses élèves dont les «colles» consistaient à participer aux travaux de fouilles les jeudis après-midi. 
Le principal acquis de ses interventions fut la publication d'un plan précis de toutes les structures visibles de son temps, le premier depuis celui qui avait été levé par F. Morel Macler (Lerat, 1956 ; Grenier, 1958, fig. 263). C'est ensuite Y. Jeannin, d'abord conservateur du musée de Montbéliard puis conservateur à la Direction régionale des Antiquités historiques de Franche-Comté, qui reprend le flambeau, de 1964 à 1984. Il est le premier à restituer les arcatures aux deux façades hémicirculaires sud et nord et à la grande façade diamétrale ouest (Jeannin, 1973 et 2002). Ses fouilles des façades sud et nord lui permettent de mettre au jour des éléments de stylobate, découverte qui entraîne l'identification d'éléments ayant appartenu aux piédroits des arcades, décorés du fameux Theatermotiv si fréquent dans l'architecture théâtrale romaine. C'est également le premier archéologue à associer systématiquement à ses fouilles des travaux de restauration et de mise en valeur des vestiges.

Depuis 2001, une équipe constituée d'archéologues et d'architectes travaille de nouveau sur l'édifice ${ }^{10}$.

Les vestiges du théâtre de Mandeure sont de deux sortes : les ruines conservées en place d'une part, un corpus d'éléments lapidaires de plusieurs centaines de blocs, d'autre part. Essayer de combiner les ruines et les éléments lapidaires n'est pas une chose aisée : beaucoup de blocs découverts au théâtre sont aujourd'hui dispersés dans les différents musées et dépôts régionaux (Mandeure, Montbéliard, Besançon, Mulhouse, etc.) et tous les blocs conservés autour du théâtre, plusieurs centaines, n'appartiennent pas à celuici. C'est dire qu'aux difficultés matérielles de manipulation en vue des rapprochements ou des collages s'ajoute l'impossibilité de pouvoir établir des critères assurés d'attribution des séries décoratives. Sans compter que, consacré d'emblée "Monument historique " et promu dans la foulée au rang de monument de référence du "théâtre gallo-romain ", le théâtre souffrit d'une notoriété précoce: maintes fois restaurés jusqu'à aujourd'hui, les murs visibles en élévation n'ont, pour la plupart, plus rien d'antique. Pourtant, le travail de récolement systématique des séries lapidaires, la réalisation de documents topographiques et de relevés architecturaux de référence, mais aussi les résultats des quelque neuf sondages ouverts depuis 2001, nous permettent de proposer les premières hypothèses de restitution du plan et de l'élévation du théâtre et de préciser la chronologie du monument.

10. Avec le soutien du Syndicat intercommunal à vocation archéologique de Mandeure-Mathay, de l'université Marc-Bloch (Strasbourg) et du DESS «Architecture et archéologie » (École d'architecture de Strasbourg).

\section{LE PLAN ET LA RESTITUTION DE L'ÉLÉVATION DU DERNIER ÉTAT}

C'est sans doute le point sur lequel nous avons fait les progrès les plus importants et les plus spectaculaires. Avant de présenter nos hypothèses, nous rappelons ici que nous proposons une reconstitution théorique et non une restitution (pour ces notions, voir Balut, 1982a et b). Les blocs ou fragments de blocs évoqués dans le raisonnement et utilisés dans le dessin peuvent appartenir, théoriquement, à chacune des trois façades à arcades du théâtre : nous ne sommes pas encore en mesure d'attribuer tel ou tel élément à une façade particulière, ni non plus, d'ailleurs, capables de faire la différence entre les blocs du premier et ceux du deuxième niveau d'arcades. Ni les modules, ni les éléments décoratifs, ni même la qualité d'exécution ne permettent aujourd'hui de faire le tri nécessaire à une véritable restitution. La faible proportion de blocs conservés, faible si on la rapporte aux quelque $250 \mathrm{~m}$ de façade en grand appareil tous niveaux confondus, et la nature de notre corpus font que nous avons fait très peu de collages ou de rapprochements entre des blocs tangents. La restitution que nous proposons ici repose par ailleurs sur l'hypothèse de l'appartenance de tous les blocs évoqués ci-dessous à l'élévation de l'édifice de spectacle. Ces prudentes réserves étant faites, les progrès faits ces dernières années du fait du traitement de la documentation ancienne, qui permet parfois de reconstituer l'histoire des éléments lapidaires depuis leur découverte grâce au relevé "pierre à pierre » des façades, nous autorisent à proposer les hypothèses qui suivent.

Le théâtre de Mandeure appartient à cette catégorie d'édifices de spectacle construits en partie sur une pente naturelle, en partie sur des maçonneries. En effet, une large partie de la cavea est adossée au talus formé par la falaise calcaire et seul le dernier quart ouest de celle-ci repose sur des substructions construites en petit appareil (opus vittatum). L'orchestra, telle qu'elle se présente, dans son état actuel, qui est aussi celui de la dernière grande phase de construction, a nécessité des travaux de creusement dans le substrat calcaire.

\section{LA CAVEA ET LE DISPOSITIF SCÉNIQUE}

La cavea se présente comme un très vaste entonnoir de $142 \mathrm{~m}$ de diamètre sur plus de $20 \mathrm{~m}$ de hauteur. Notons le caractère exceptionnel de la dénivellation : au théâtre d'Autun (Saône-et-Loire), le seul à présenter un diamètre supérieur à celui de Mandeure, il n'y a «que » $12 \mathrm{~m}$ de 


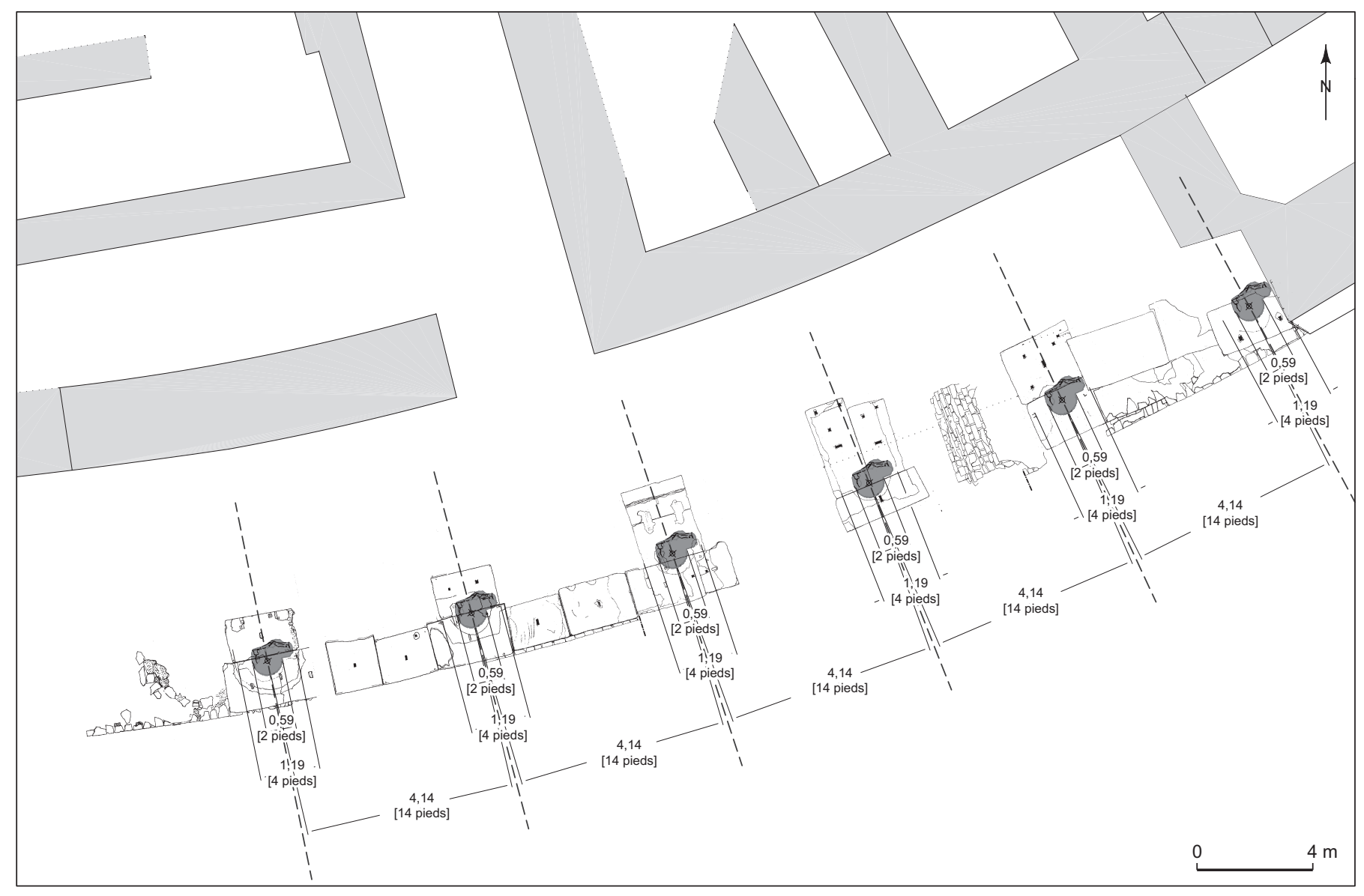

- - - entraxe des piliers du $1^{\mathrm{er}}$ niveau

bloc B0026

relevé de détail (échelle : $1 / 10$ )

relevé topographique Chazaly, 1999

Fig. 23 - Relevé de la façade sud du théâtre de Mandeure (DAO : N. André).

différence entre le niveau de l'orchestra et celui qui correspond au sommet du dernier maenianum. Les gradins sont répartis en quatre maniana, d'inégale hauteur et ne présentant pas les mêmes pendages. Ceux-ci sont séparés tantôt par des ambulacres, tantôt par des couloirs de précinction. Des substructions en opus vittatum, plus nombreuses du côté sud que du côté nord, complètent le demicercle vers l'aval. Comme il se doit dans un théâtre romain, elles sont couvertes de voûtes rampantes. Certaines ont été remblayées et forment de simples caissons, d'autres accueillent des escaliers, des couloirs ou des vomitoria. Les gradins ont tous disparu. Seules les fondations du premier maenianum sont conservées : elles soutenaient presque certainement des gradins en pierre. Il n'est pas impossible, en revanche, que les gradins des media et suma cavea aient été en bois. L'étude des circulations, qui permettaient de répartir les quelque 18000 spectateurs, est loin d'être achevée et n'est guère facilitée par la disparition complète des niveaux de circulation antique. Le nombre des circuits possibles et leur implantation dans le plan d'ensemble de l'édifice nous permettent de faire néanmoins l'hypothèse d'un dispositif très "classique ", organisant les accès en fonction des statuts des spectateurs et, bien sûr, des emplacements réservés aux uns et aux autres (Kolendo, 1981 ; Ville, 1982).

Les dimensions très réduites de l'orchestra, au vu en tout cas de l'ensemble de l'édifice (13,40 $\mathrm{m}$ de diamètre), ne laissent pas d'étonner. Elle résulte probablement d'un aménagement assez tardif, intervenu à un moment où il fallait bien se contenter de l'espace disponible. Retenons qu'elle présentait un beau dallage de plaques de calcaire et que leur forme de demi-cercle outrepassé (de $2 \mathrm{~m}$ ) trouve 


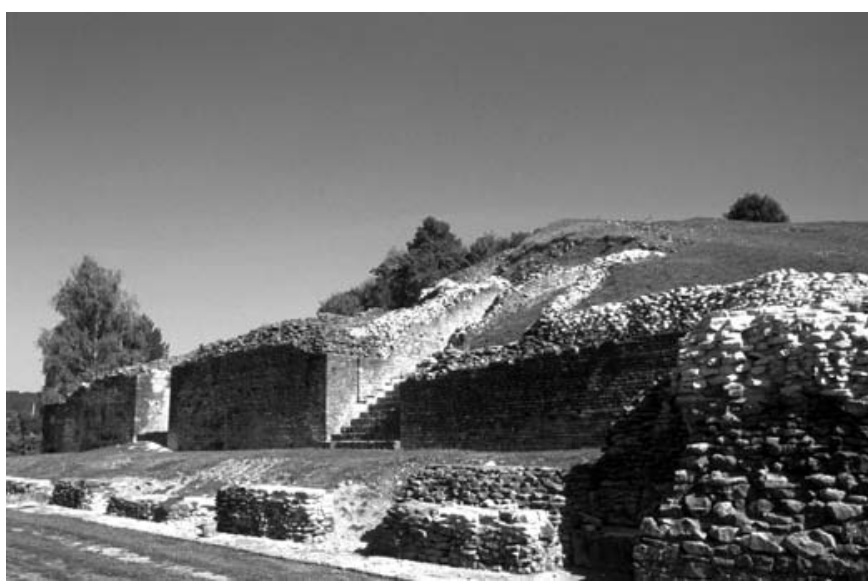

Fig. 24 - Vue de la façade ouest du théâtre de Mandeure (cliché : J.-Y. Marc, Université Marc-Bloch).

de nombreux parallèles dans l'architecture théâtrale des provinces gauloises : Alésia (Côte-d'Or), Drevant (Cher), Jublains (Mayenne), Sanxay (Vienne), Bouzy-la-Forêt (Loiret), Valognes (Manche), etc.

Le pulpitum a été dégagé au moins deux fois depuis les fouilles de F. Morel-Macler, mais n'a fait l'objet ni de plan ni de description précise $(9,30 \mathrm{~m}$ x 3,10 m). Il était flanqué de chaque côté, au-delà d'un hydragogue qui évacuait les eaux de l'orchestra vers la plaine du Doubs, de deux pièces rectangulaires et aveugles. Ces deux paraskenia, dont la fonction et l'usage restent inconnus, devaient présenter une élévation particulièrement imposante, si l'on en juge par l'épaisseur de leurs maçonneries et la puissance de leurs fondations ${ }^{11}$.

\section{LES FACADES}

Les façades sont inégalement parvenues jusqu'à nous. La façade sud conserve encore son assise de stylobate sur $22 \mathrm{~m}$ environ, correspondant à cinq travées (entraxe : 4,14 m). Celle-ci est constituée de blocs d'opus quadratum, solidement cramponnés les uns aux autres et dont les parements sont grossièrement ravalés. Sa conservation partielle nous a permis d'analyser précisément les traces laissées par l'implantation des éléments d'élévation : des piédroits décorés de demi-colonnes engagées (fig. 23). On peut donc établir le rythme des supports indépendants et restituer huit travées jusqu'à l'angle de cette façade. Ce rythme correspond exactement à celui que l'on peut

11. Voir Coupel, Frézouls, 1956 ; Courtois, 1989. restituer sur la façade nord, à partir de l'observation des vides laissés par la récupération des blocs de grand appareil. Sur la grande façade ouest, le même système de puissants supports indépendants est attesté sauf au niveau de l'orchestra (fig. 24) ; le rythme est en revanche légèrement plus lâche (entraxe : 4,32 m).

Rien que pour ce premier niveau, on peut d'ores et déjà restituer trente-cinq travées : huit du côté sud, sept du côté nord et deux fois dix du côté ouest. Y. Jeannin avait déjà reconnu côté sud les premiers blocs d'un deuxième niveau de stylobate. Il faut donc restituer un deuxième niveau de supports indépendants, au moins sur les deux façades hémicirculaires.

L'important travail réalisé sur le lapidaire, rangement, classement, enregistrement et relevé, nous a permis de commencer à combiner ces données avec des éléments d'élévation en grand appareil ${ }^{12}$. La connaissance du diamètre, parfaitement mesurable sur les éléments conservés en place du stylobate de la façade sud, nous a permis de retrouver des fragments de fûts de demi-colonnes engagées. L'attribution aux façades du théâtre d'un nombre non négligeable de claveaux imposait, par ailleurs, la restitution d'arcades. Enfin, les importants progrès réalisés dans l'attribution des éléments lapidaires grâce à l'examen attentif de la très abondante documentation photographique de nos prédécesseurs, nous autorise à compléter avec beaucoup de certitude le Theatermotiv des grandes façades du théâtre de Mandeure. Étant donné que ce travail est loin d'être achevé, nous nous contenterons de commenter, dans les lignes qui suivent, l'hypothèse de restitution d'un piédroit du premier niveau d'arcades (fig. 25, $\mathrm{n}^{\circ} 1$ ).

La demi-colonne engagée reposait sur une base sans plinthe, constituée de l'alternance classique de deux tores et d'une scotie. Les fûts présentaient des cannelures rudentées jusqu'au sommet. Nous avons la chance d'avoir trouvé un fragment de ces fûts présentant à droite le départ d'un arc (c'est ce bloc qui permit à Y. Jeannin de proposer, le premier, de restituer une façade à arcades). Plusieurs claveaux de ces arcs ont été retrouvés : décorés de deux fasces en parement, ils n'étaient pas extradossés. Aucun chapiteau ne peut pour le moment être attribué avec certitude à cette série. Les éléments d'entablement, en revanche, ne manquent pas. Les éléments d'architrave, décorés en parement de trois

12. C'est pour nous l'occasion de remercier C. Mougin, auteur d'un mémoire de Maîtrise sous la direction de H. Walter (Mougin, 1997), qui a mis à notre disposition l'ensemble de sa documentation et a participé aux deux premières campagnes d'étude des vestiges architecturaux. 


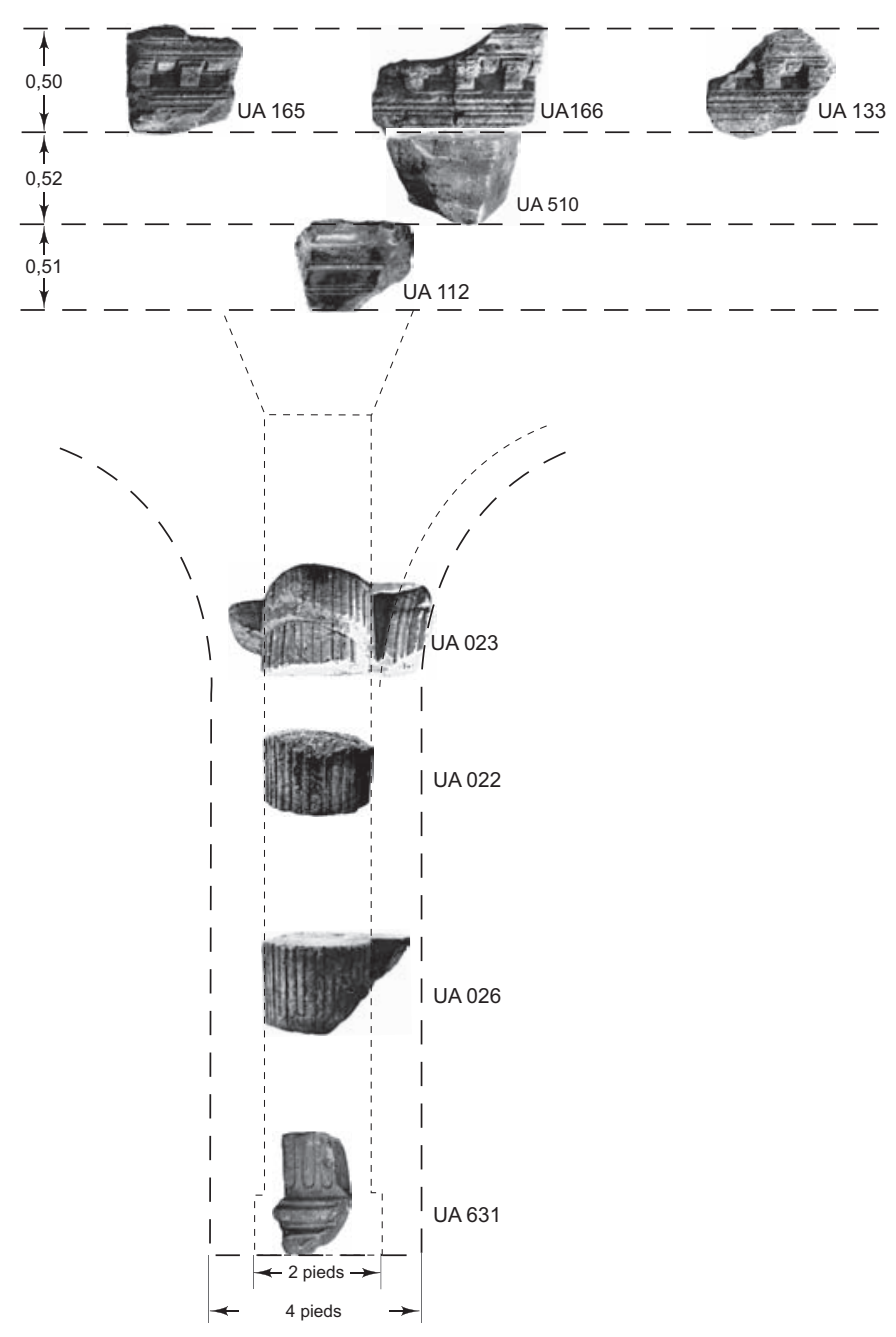

1

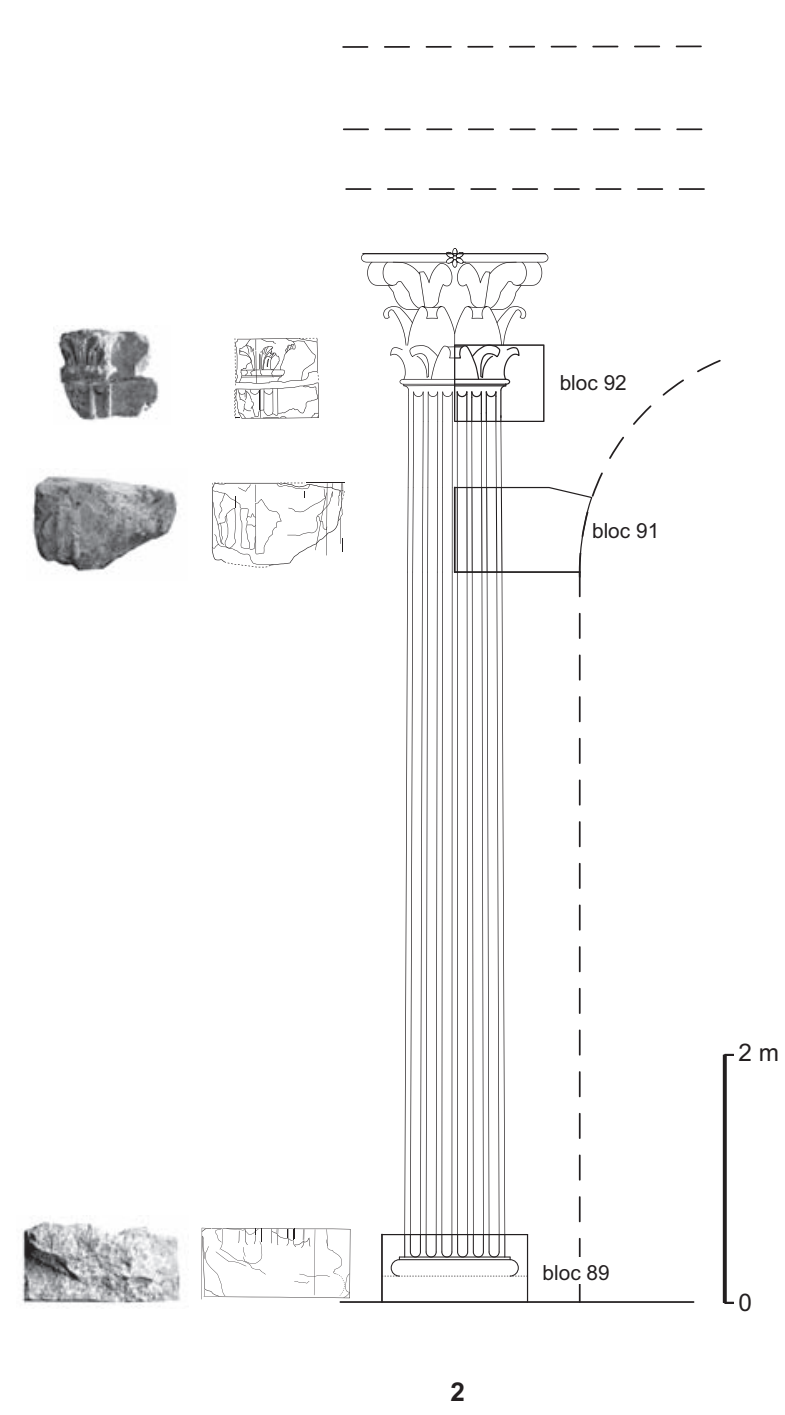

2

Fig. 25 - Théâtre de Mandeure : 1, restitution de l'élévation du premier niveau d'arcades; 2, restitution d'une arcade à partir de trois fragments découverts dans les fouilles du gazoduc réalisées en 1984 par Y. Jeannin (DAO: $n^{\circ} 1$, S. Blin; $n^{\circ} 2$, N. André).

bandeaux talutés et de hauteur croissante, sont les plus abondants. En revanche, un seul bloc de frise a pu être à ce jour identifié ; il montre que cette assise se présentait sous la forme d'une plate-bande clavée. Enfin, de nombreux fragments de blocs de corniche modillonnaire à moulures lisses découverts au théâtre peuvent être proposés pour le dernier niveau de l'entablement.

Nous proposons de restituer le même schéma au deuxième niveau. Le troisième niveau en revanche présentait un motif architectural différent. Il s'agit cette fois d'un ordre à ressauts soutenu par des pilastres. C'est ce que suggère la découverte d'au moins deux fragments de frise architravée à décrochement. Il s'agit de blocs présentant, dans un même développement décoratif, un motif d'architrave à deux fasces, séparées par une baguette et couronnées d'un talon surmonté d'un bandeau vertical, et un motif de frise lisse dans la partie supérieure. La restitution de ce troisième niveau est corroborée par la présence d'un chapiteau toscan de pilastre dans le lapidaire conservé autour du théâtre, qui présente un ressaut de dimensions équivalentes à celles mesurées sur les blocs décrits ci-dessus.

On le voit, ce ne sont pas seulement les dimensions qui font du théâtre de Mandeure un édifice exceptionnel dans l'architecture des Gaules et des Germanies : c'est également le traitement très "classique" des façades. Cette «monumentalité proclamatrice», pour reprendre une formule utilisée très récemment à l'amphithéâtre d'Avenches (Bridel, 2004, p. 215-220), dénote la préten- 
tion de cette architecture séquane qui s'est exprimée aussi bien dans sa capitale, Vesontio, que dans les autres grandes agglomérations de la cité, Villards-d'Héria et, donc, Mandeure.

\section{PHASES DE CONSTRUCTION ET DATATION}

L'ouverture ces trois dernières années de neuf sondages ne nous a pas permis de répondre à tous les objectifs fixés au départ, en particulier ceux qui concernaient l'histoire de l'édifice. La maigreur des résultats en ce qui concerne la chronologie absolue, qui s'explique dans une très large mesure par l'indigence du matériel découvert et la quasiabsence de fossiles chronologiques fiables, nous empêche, pour le moment, de proposer des hypothèses historiques sur les différentes phases de construction et d'utilisation de l'édifice de spectacle. Nous avons quelques raisons de penser que la date proposée par Y. Jeannin pour la construction du théâtre, l'époque flavienne, n'est pas établie avec certitude et, qu'en tout état de cause, cette datation ne concerne qu'une des phases de construction et probablement pas celle de la façade à arcades qui est actuellement visible.

En revanche, nous avons été plus heureux en matière de chronologie relative. Y. Jeannin avait déjà proposé l'hypothèse de deux grandes phases de construction, avec une transformation importante de l'orchestra dans la seconde phase. Les observations que nous avons pu faire ont largement confirmé ses hypothèses. Nous avons en effet cru pouvoir montrer que le théâtre de Mandeure présente probablement trois grandes phases de construction, que l'orchestra dans sa forme actuelle appartient à la dernière et qu'auparavant elle pu être plus grande et plus haute, qu'il n'est pas exclu qu'une arène ait été aménagée à cet endroit. Cette séquence historique qui voit un théâtre succéder à un édifice à arène pourrait passer pour extravagante si le site voisin d'Augusta Raurica ne présentait pas la même succession (voir dernièrement: Horisberger, 1997). Plus que sur l'histoire de l'édifice de spectacle, suspendue aux recherches en cours, c'est sur l'appréciation de sa décoration architecturale, celle de sa dernière grande phase monumentale en tout cas, que nous souhaitons insister ici.

\section{LA DÉCGORATION ARCHITECTURALE}

Le très grand nombre de blocs architecturaux ou de fragments lapidaires isolés provenant de Mandeure atteste l'existence de programmes décoratifs riches et originaux. La situation n'est certes pas comparable à celle de Saintes (Charente-Maritime), de Narbonne (Aude) ou de Périgueux (Dordogne), pour prendre trois exemples étudiés récemment, ni même à celle d'Autun (Saôneet-Loire) ou de Langres (Haute-Marne), deux capitales voisines où d'ambitieux programmes architecturaux ont laissé de très importantes séries lapidaires. Toutefois, toutes proportions gardées, notre collection lapidaire témoigne également du vaste naufrage de l'architecture monumentale de l'antique Epomanduodurum. Malheureusement, ces vestiges lapidaires ont été dispersés au gré des péripéties de l'histoire des fouilles et répartis entre divers dépôts. Les collections lapidaires du musée des Beaux-Arts et d'Archéologie de Besançon et du musée d'Archéologie de Montbéliard conservent une grande partie des fragments architecturaux (environ 150 blocs en marbre ou en calcaire) représentatifs des fouilles réalisées au XIX ${ }^{\mathrm{e}} \mathrm{s}$., tandis qu'à Mandeure même, on trouve plus de 600 fragments provenant de fouilles plus récentes, mais conservés, ou déposés, dans plus de quatre lieux différents, sans parler, bien sûr, des nombreux blocs remployés dans les maisons de la ville moderne.

Cette collection s'est encore enrichie récemment de nouvelles découvertes, grâce surtout aux fouilles des fortifications du Bas-Empire, avec une «récolte» de plusieurs centaines de fragments de blocs, ou aux sondages ouverts dans le théâtre. Un effort de rangement, d'enregistrement et de classement de tous ces éléments a été entrepris dès la reprise des recherches sur le théâtre; effort qui doit être prolongé dans les prochaines années par la construction d'un petit pavillon de site, destiné à protéger et présenter une partie au moins de ce très riche corpus lapidaire, sans aucun doute l'un des plus riches de la région. En attendant, les difficultés inhérentes à l'« émiettement » et à la dispersion de la collection, le temps qu'il a fallu pour se faire une idée de son ampleur et pour en étudier les principales caractéristiques, font qu'il est impossible, dans les lignes qui suivent, de proposer autre chose que des hypothèses liminaires.

Comme nous l'avons déjà dit, nous ne disposions, au moment où nous avons repris ce travail, de presque aucune attribution fiable. Cette situation nous imposait par conséquent de traiter le corpus lapidaire dans son ensemble, sans chercher à classer les différents éléments en les attribuant à des édifices dont nous ignorions à peu près tout, à l'exception bien sûr du théâtre, le seul élément de la panoplie monumentale à être parvenu jusqu'à nous. C'est pourquoi ces séries lapidaires sont étudiées sous deux aspects. Elles sont, tout d'abord, des éléments essentiels des hypothèses de restitution des élévations des vestiges connus (théâtre, sanctuaire, thermes, etc.) : c'est l'étude de leurs caractéristiques 


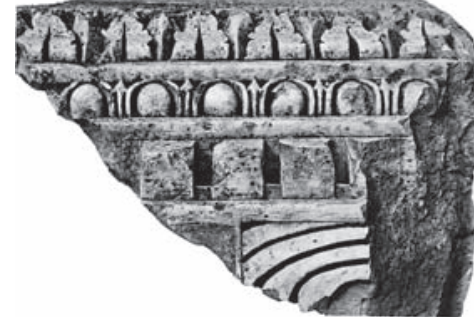

0 $20 \mathrm{~cm}$

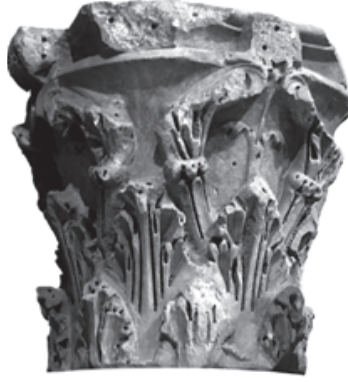

2
Fig. 26 - Théâtre de Mandeure : 1, corniche modillonnaire en calcaire conservée au théâtre; 2, chapiteau corinthien en marbre blanc conservé au musée des Beaux-Arts et d'Archéologie de Besançon (clichés : S. Blin).

métrologiques et architectoniques qui permet de proposer une élévation au théâtre. Elles sont par ailleurs envisagées dans le cadre de la production des ateliers de lapicides dans le nord-est des Gaules et les Germanies. La reconnaissance de ces ateliers nécessite le rassemblement d'un corpus de formes décoratives, qui est encore très largement méconnu. Depuis l'ouvrage de H. Kähler sur les chapiteaux corinthiens de la région rhénane et hormis les synthèses remarquables qui portent sur les cités helvètes et trévires ${ }^{13}$, les recherches restent rares dans cette région. Depuis les études réalisées sur Saintes, Périgueux ou Alésia, c'est devenu un lieu commun que de pointer du doigt ce manque de publication en Gaule romaine ${ }^{14}$. Dans ce contexte, les productions manduriennes, par leur qualité et leur abondance, présentent un intérêt tout particulier.

Bien que certaines séries émergent résolument de la collection et permettent déjà de proposer une évolution générale des formes décoratives sur le site, les calages chronologiques sont, pour l'instant, peu assurés. La pauvreté des indices stratigraphiques réduit les propositions de datation aux seules conjectures stylistiques qui sont, dans l'est des Gaules et les Germanies, comme on l'a dit précédemment, en cours d'élaboration. Le vocabulaire architectural de la période augustéenne est, par exemple, complètement inconnu, alors qu'on connaît bien les témoignages de cette période à Autun (Olivier, 1985) ou à Langres (Joly, 2003, p. 233). À Mandeure, les premiers programmes décoratifs qui sont pour l'instant attestés sont réputés contemporains de la période flavienne.

13. Kähler, 1939. Pour Augst: Bossert-Radtke, 1992 ; Trunk 1994. Pour Avenches : Bridel, 1982 ; Bossert-Radtke, 1992. Pour Neumagen : Numrich, 1997

14. Pour Alésia: Olivier, 1989. Pour Saintes: Tardy, 1989, 1990 et 1995.
Parmi les nombreux édifices qui composaient la panoplie monumentale de Mandeure, celui qui possède l'élévation et la décoration les mieux connues est le théâtre. Il présente des choix décoratifs simples et de facture très soignée. L'entablement est composé d'une architrave à trois fasces talutées et de hauteur croissante, dont les transitions sont assurées par des moulures lisses. Le même motif est repris sur les parements des claveaux. L'architrave est surmontée d'une frise lisse et de corniches modillonnaires à moulures lisses (fig. 25, $\mathrm{n}^{\circ} 1$ ). On retrouve ce type de corniche au Capitole de Besançon (Castan, 1986), ou au théâtre et au sanctuaire thermal de Moritasgus d'Alésia (Olivier, 1989). L'ensemble, qui correspond bien aux cartons italiens, s'inscrit dans des modèles décoratifs romains "classiques".

D'autres types de corniches modillonnaires à moulures et métopes ornées de palmettes, qui ne sont pas attribuées au théâtre, présentent également un vocabulaire esthétique très «classique ". Sur ces dernières sont repris, avec plus ou moins d'élégance et d'habileté selon les mains, un motif végétalisé de rais-de-cœur en ciseau, un registre d'oves et de pointes de flèche surmonté d'un registre de denticules, dont les viae sont taillés en sifflet (fig. $26, \mathrm{n}^{\circ} 1$ ). On peut supposer que ces corniches proviennent du sanctuaire, de même qu'une série de blocs comprenant une base de pilastre, un fût de pilastre avec départ d'arc et un chapiteau de pilastre, dont le rapprochement a déjà permis de restituer une arcade (fig. 25, $\mathrm{n}^{\circ} 2$ ).

À ces entablements, on peut associer des chapiteaux corinthiens, en assez petit nombre d'ailleurs, qui attestent eux aussi l'influence des cartons méditerranéens. On note ainsi un exemplaire en marbre blanc, actuellement conservé au musée des Beaux-Arts et d'Archéologie de Besançon (fig. 26, $\mathrm{n}^{\circ}$ 2). Il présente deux couronnes de feuilles d'acanthe aux lignes d'ombres très marquées, notamment au niveau de la nervure axiale. On retrouve le même traitement au niveau des caulicoles légèrement divergents du registre supérieur. Ils sont parcourus de profondes incisions verticales et décorés d'un collier de sépales inversés. Du collier s'échappe un calice bipartite, la feuille externe soutenant la base de la volute et la feuille interne soutenant la crosse médiane. Les moulures de l'abaque sont laissées sans ornement. Selon H. Kähler, ce type de chapiteau que l'on trouve aussi à Trèves, à Mayence ou à Magdebourg, est un témoignage du travail des lapicides méditerranéens dans la région (Kähler, 1939, p. 82-86).

Les séries lapidaires de Mandeure présentent par ailleurs des caractéristiques formelles qui les rapprochent des séries plus locales, celles de la vallée rhénane ou de l'arc jurassique. 

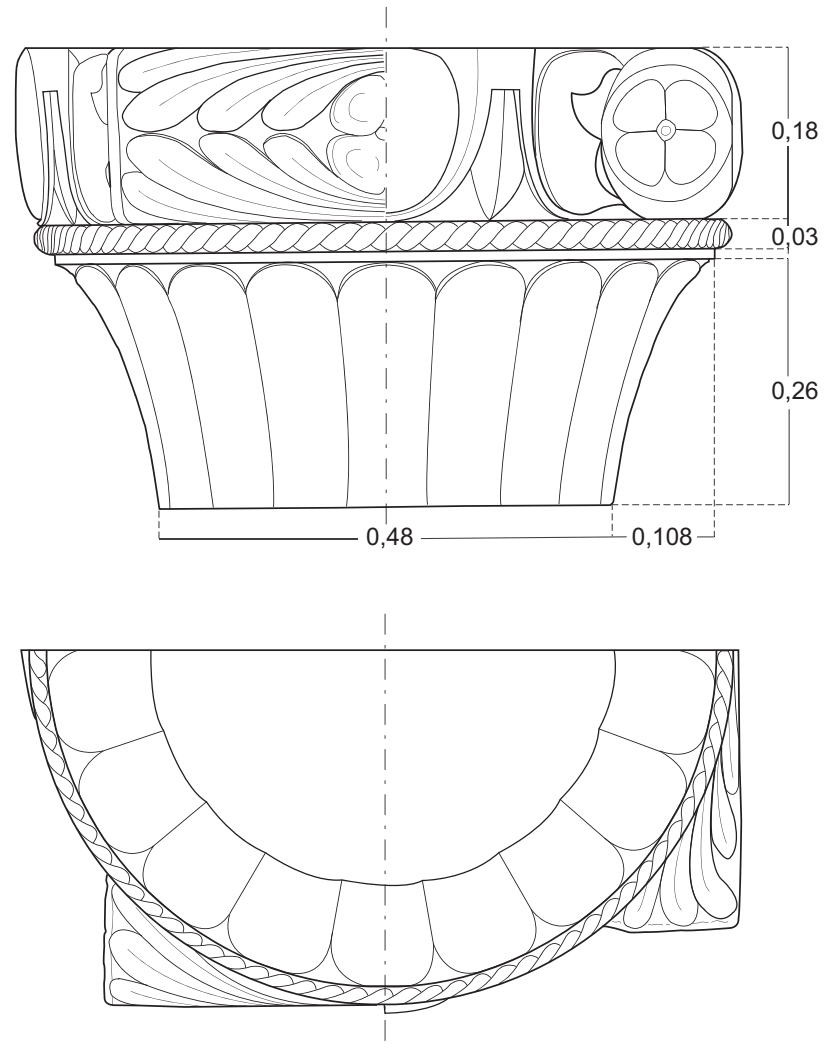

1
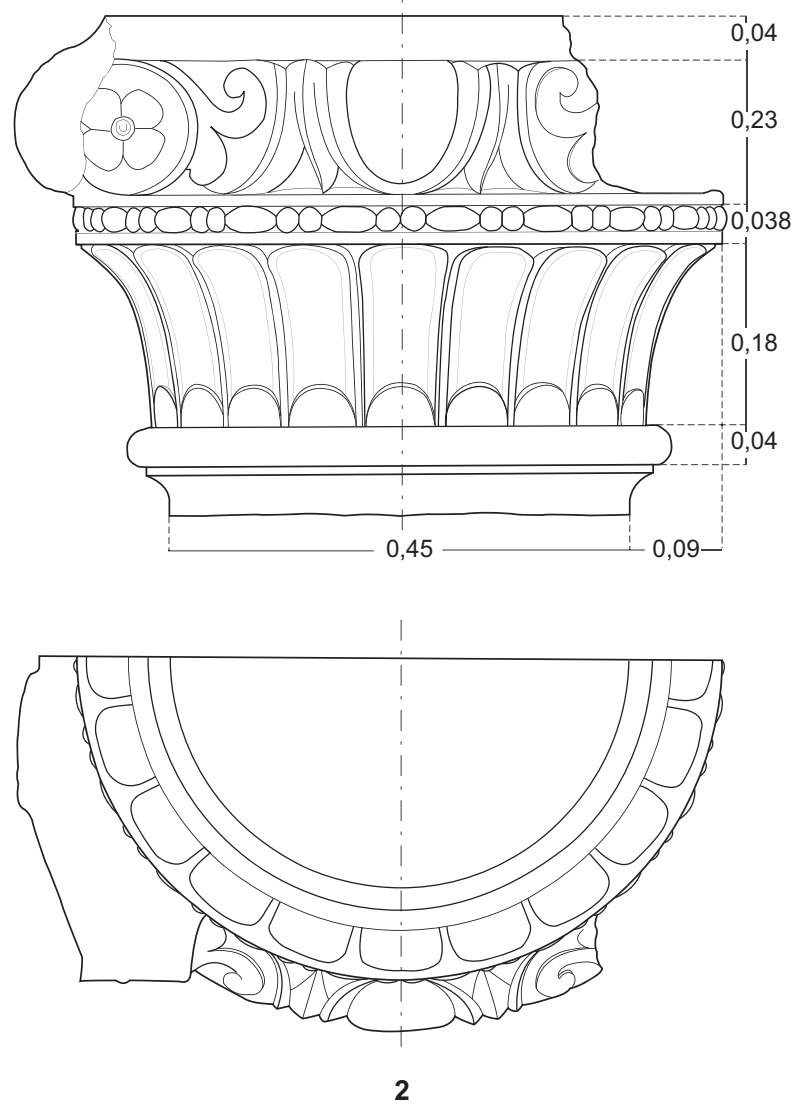

Fig. 27 - Chapiteaux composites en calcaire (DAO : S. Blin, d'après F. Morel-Macler, Supplément aux Antiquités de Mandeure, folio 16).

On peut d'ores et déjà établir des rapprochements intéressants entre les séries séquanes et helvètes, par exemple. Un chapiteau corinthien en calcaire découvert à Mandeure et conservé aujourd'hui au musée d'Archéologie de Mulhouse est très proche d'une série découverte à Nyon et attribuée à la basilique de cette ville ${ }^{15}$. Les caractéristiques des ateliers rhénans se trouvent également représentées par plusieurs chapiteaux composites ionicisants ${ }^{16}$. Les motifs employés sur le calathos (motifs de feuilles d'eau ou de file de godrons, avec ou sans lunule) ou sur les balustres révèlent, par la diversité des modèles décoratifs représentés, un grand nombre de variantes typologiques (fig. 27). Ces chapiteaux, très répandus dans la région, se retrouvent durant tout le Haut-Empire depuis Trèves jusqu'à Autun en passant par Augst et Kaiseraugst.

15. Musée de Mulhouse, nº d’inv. : 2009 (Kähler, 1939, type no 32).

16. Kälher (1939) regroupe ces chapiteaux composites sous la dénomination de "chapiteaux à calathos de godrons" (Kapitelle mit Pfeifenblattkalathos). Celle-ci est reprise par Trunk, 1994, p. 120-125. On préférera celle qui a été proposé par Tardy (1989) qui, en se fondant sur la présence des balustres, les associe plus justement à une forme dérivée du ionique normal.
Ceux qui sont conservés à Mandeure présentent toutefois des formes assez tardives, comparables aux chapiteaux découverts à Kaiseraugst qui sont datés du $\mathrm{III}^{\mathrm{e}} \mathrm{s}$.

Les caractéristiques formelles des chapiteaux toscans découverts à Mandeure sont plus difficiles à déterminer. Ils présentent en effet un handicap majeur : la succession répétitive des moulures (cavet, doucine, filet) ne permet guère de mettre en évidence des critères stylistiques, ce qui rend ainsi difficile toute tentative de chronologie relative. À Mandeure par exemple, on constate l'association cavetdoucine qui est fréquemment représentée ailleurs en Gaule romaine (fig. 28). Cette association domine également sur les profils des chapiteaux de la villa de Plassac en Gironde (Bost, Monturet, 1987), de Saintes (Tardy, 1989, p. 139-150), de Haute-Savoie (Broise, 1969) ou encore de Besançon. On trouve aussi à Mandeure des compositions plus inhabituelles, avec des profils qui associent parfois deux ou trois moulures simples (cavet). P. Broise, après A. Grenier, avait soulevé le problème posé par l'étude de ces chapiteaux du type «toscan provincial » et les difficultés de son apprécia- 


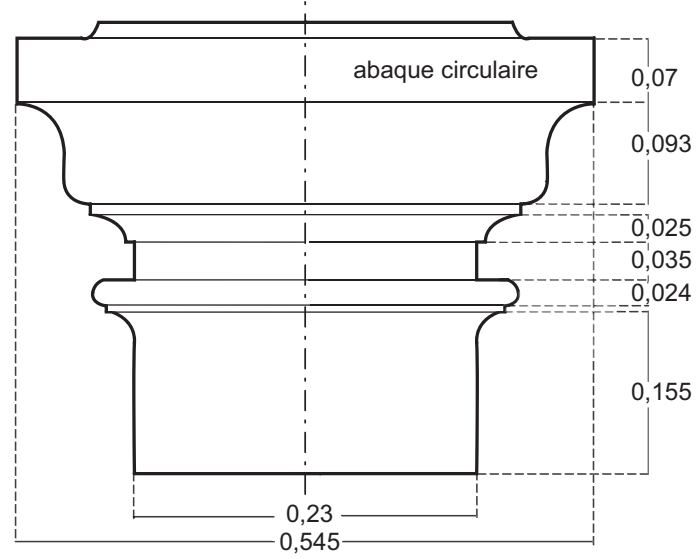

1

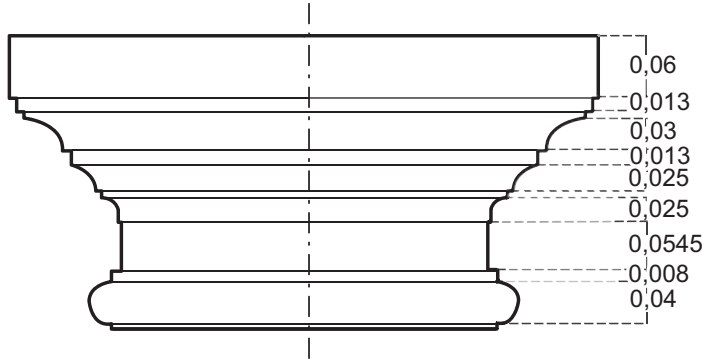

3

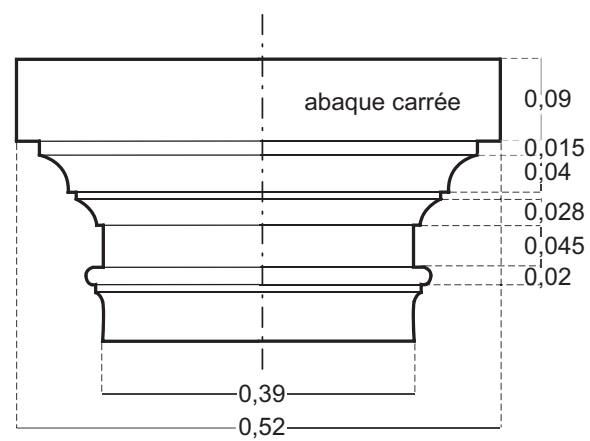

2

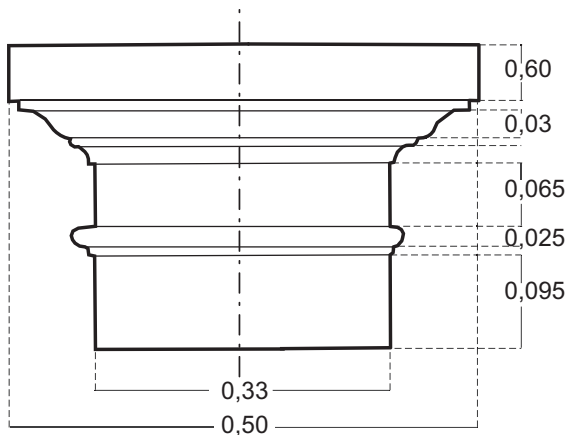

4

Fig. 28 - Chapiteaux toscans en calcaire : 1, 2, chapiteaux découverts au lieu-dit En Coudroie; 3, chapiteau découvert en 1859 au nord du village près du Doubs ; 4, chapiteau découvert au-dessus du théâtre de Mandeure (DAO : S. Blin, d'après F. Morel-Macler, Supplément aux Antiquités de Mandeure : $n^{\text {os }} 1$ et 2, folio $18 ; n^{\circ} 3$, folio $19 ; n^{\circ} 4$, folio 7 ).

tion chronologique, et souligné la nécessité de préciser leur évolution par des inventaires plus répandus des "dépôts locaux »(Grenier, 1958, p. 41 ; Broise, 1969). Plus de trente ans après, la situation n’a guère changé.

L'étude stylistique des séries lapidaires ne fait que débuter. Mais nos premières recherches font apparaître une série de caractéristiques, dans lesquelles on reconnaît aussi bien les influences des ateliers de l'Urbs que celles des productions «rhénanes». Il est impossible, pour l'instant, de postuler l'existence d'ateliers «manduriens", mais les observations et les comparaisons qui peuvent déjà être réalisées en Séquanie et dans les régions voisines témoignent d'une certaine communauté avec, bien sûr, les programmes de la capitale de cité, Besançon, mais aussi avec les grands programmes architecturaux d'Avenches, de Nyon ou d'Augst.

\section{LE THÉÂTRE, SON CONTEXTE URBANISTIQUE ET LA PANOPLIE MONUMENTALE D'EPOMANDUODURUM}

L'étude du théâtre antique s'est très vite enrichie d'une recherche plus générale sur la panoplie monumentale de l'agglomération antique. Sa position en face d'un très grand sanctuaire, position qui lui valut d'être considéré comme un élément même de ce sanctuaire, exigeait de sortir d'une approche strictement monographique. Par ailleurs, la seule analyse de ses évolutions morphologiques ne permettait pas de comprendre la place de l'édifice dans le paysage urbain ou le type de relation qu'il était censé entretenir avec la communauté. Ces remarques s'imposent d'autant plus que l'édifice, par la hauteur de ses façades et son imposante masse architecturale, devait être visible d'à peu près partout 
dans la ville antique et constituer, probablement, le repère monumental majeur du paysage urbain.

Les prospections menées depuis 2001 sur l'ensemble de l'agglomération ont considérablement enrichi la parure monumentale de la ville antique, en particulier dans le secteur du théâtre qui se voit désormais doté d'une riche série d'édifices publics (fig. 4 et pl. X, hors texte). Comme à Avenches, l'esplanade qui séparait le sanctuaire de l'édifice de spectacle est encadrée par toute une série de constructions qui ont été révélées aussi bien au sud qu'au nord du théâtre. Seule une fouille permettra de les identifier plus précisément, mais on devine déjà des lieux de cultes secondaires ou des édifices nécessaires pour l'accueil ou le confort des pèlerins. D'autres grands bâtiments se laissent entrevoir dans le centre de l'agglomération, dans la courbe du Doubs par exemple. Ce constat d'un riche équipement architectural, que l'on pouvait déjà faire au vu des découvertes anciennes par F. Morel-Macler d'un lapidaire abondant attestant l'existence de plusieurs constructions monumentales, a donc été confirmé par les prospections réalisées depuis trois ans. Si l'on ajoute à ces observations que les premières études menées sur la décoration architecturale ont établi qu'il faut restituer aux monuments publics une décoration architecturale riche et soignée, on conviendra que nous avons probablement affaire avec ce site à une métropole urbaine qui présente tous les attributs de l'urbanitas romaine. Reste à ajouter que le théâtre a probablement joué un rôle directeur dans la mise en place de ce paysage monumental: on a pu en effet remarquer que bon nombre des édifices repérés en prospection, à proximité du théâtre bien sûr mais également dans la courbe du Doubs, sont alignés sur celui-ci, comme si l'équipement architectural s'était trouvé aimanté en quelque sorte par l'édifice de spectacle.

Ces premiers résultats confirment pleinement l'impression que donnaient déjà, outre les dimensions considérables de la ville antique, 180 ha urbanisés, le grand sanctuaire à péribole ovale et temple périptère sine postico, l'établissement thermal de Courcelles (Mougin, Watts, 1997), l'inscription évergétique de Catullus (CIL, XIII, 5416 a et b, et 5417), la présence du culte impérial, la statue de Mars (Pichot, 1995a et b), etc. : Epomanduodurum était une importante ville gallo-romaine. Il n'est pas question de discuter ici du statut de cette ville. Mandeure était, au sens juridique du terme, une agglomération secondaire, mais son épiderme monumental et son importance dans la géographie urbaine de la région étaient, quant à eux, loin d'être secondaires. Il convient d'insister sur ce point pour s'inscrire en faux contre une synthèse publiée très récemment qui pourrait laisser penser que Mandeure n'était qu'une simple bourgade à peine dotée des éléments de composantes urbaines (Walter, 2003). Un rapide coup d'œil aux plans les plus récents de la ville antique ou même une simple visite sur le site du théâtre suffisent à démentir complètement de telles conclusions, il est vrai, écrites avant le début de nos travaux.

\section{CONCLUSION}

La reprise des recherches sur le théâtre antique de Mandeure, recherches qui se poursuivront dans les années qui viennent pour aboutir à une publication monographique, s'inscrit dans un contexte de reprise des études sur les théâtres antiques des provinces de Gaule ou de Germanie (Dumasy, 2000). Les édifices de spectacle d'Augst et d'Avenches, pour ne citer que deux exemples très proches géographiquement de Mandeure, ont fait l'objet de travaux importants ces dernières années ${ }^{17}$. Il était temps car le débat sur la forme architecturale du théâtre gallo-romain et sa fonction commençait à «tourner en rond » (Dumasy, 1989). On sait que la plupart des édifices de spectacle ont souvent été fouillés avant la Première Guerre mondiale, la grande majorité entre 1850 et 1900 . C'est dire que la documentation ne s'est guère renouvelée depuis A. de Caumont, le premier qui regroupa les théâtres des provinces du nordouest de l'Empire dans cette catégorie à la fois topique et fonctionnelle. Depuis les années 1960, les fouilles ou la reprise des dégagements des théâtres d'Alésia, de Grand, de Ribemont-sur-Ancre (Cadoux, 1992), de Canouville (Le Maho, Nicolle, 1979), de Vendeuil-Caply (Dufour, 1992), de Lenzburg (Niffeler, 1988), de Ladenburg (Filzinger dir., 1976) ou de Dalheim (Krier, Wagner, 1985), attestent certes un regain d'intérêt pour les édifices de spectacle dans les Gaules et les Germanies, mais on déplore toujours l'absence de véritables publications monographiques.

J.-Y. M., P. M., S. B., N. A.

\section{LA FORTIFICATION DU BAS-EMPIRE}

Notre premier bilan ne repose qu'en partie sur les acquis de deux campagnes de fouilles, réalisées dans le cadre du PCR en 2002 et 2003. Ils constituent, en fonction du programme du PCR 2001-2004, avant tout les résultats tirés de l'analyse et de l'exploitation d'un grand nombre de données et d'objets récoltés au fil des années par de

17. Pour Augst: Schwarz, 1991 et Horisberger, 1997 (avec la bibliographie des dernières campagnes). Pour Avenches : Matter, 1999 et Bridel, 2004. 